\section{Yellow flowers to order}

Any well-stocked florist has no dearth of colors to pick from when putting together a bright bouquet. But for some of the most common ornamental flowers, such as geraniums, cyclamens and morning glories, yellow color varieties remain conspicuous by their absence. This situation has been rectified by Ono et al., who have succeeded in characterizing the final enzyme step in the biosynthesis of a class of flavonoids, the aurones, that confer a bright yellow hue to, among others, snapdragon flowers (Antirrhinum majus). Coexpression of this enzyme, a chalcone $4^{\prime}-0$ glucosyltransferase, and a previously described enzyme, aureusidin synthase, dramatically increases levels of aurones in the wishbone flower (Torenia hybrida). When the biosynthesis of anthocyanins, a class of flavonoids responsible for blue coloration, is simultaneously downregulated by RNA interference, bright yellow flowers result (see inset picture). The authors propose that the use of watersoluble aurones adds a new dimension to engineering the color of ornamental flowers, complementing what can be achieved using more traditional, hydrophobic carotenoid pigments. (Proc. Natl. Acad. Sci. USA 103, 11075-11080, 2006)

\section{RNA aptamer gymnastics}

RNA aptamers can display a range of affinities for their ligands, and attempts to improve binding have focused on interactions between the two molecules. However, as RNA can assume complex structures, intramolecular interactions might affect ligand binding as much or more than intermolecular binding. Carothers et al. investigate this possibility by analyzing the binding characteristics of 11 GTP-binding aptamers selected from a library of random sequences. In competition-binding assays, they compare aptamer-binding affinities to GTP with binding to 16 different GTP analogs — 10 with bulky substitutions to determine the importance of proximity between aptamer and ligand, and 6 in which a functional group was removed or modified to probe various bonds. They found that modifications to the nucleobase had a greater effect on binding than those to the ribose or phosphate. Each aptamer had a unique profile of interactions with regions of GTP. Surprisingly, they found that all aptamers, regardless of binding strength, reacted with the same number of analogs; those with tighter binding did not preferentially bind to GTP. The authors hypothesize that the stability of the aptamer's secondary structure $\left(\Delta \mathrm{G}_{\text {folding }}\right)$ may play a role. In support of this, they found a correlation between $\Delta \mathrm{G}_{\text {folding }}$ and the binding constant of the aptamer-ligand combination. (J. Am. Chem. Soc. 128, 7929-7937, 2006)

\section{Stress linked to tumor angiogenesis}

Until now, stress has been implicated in the promotion of tumor growth mostly by evidence that it represses the immune response to tumor cells. But Thaker et al. demonstrate that chronic stress can also directly promote tumor angiogenesis and growth without the involvement of the immune system. After designing a physical restraint system in which repeated immobilization triggers the release of chronic stress-associated hormones, such as norepinephrine, the authors show that tumor growth

Research Highlights written by Laura DeFrancesco, Peter Hare, Teresa Moogan, Gaspar Taroncher-Oldenburg \& Jan-Willem Theunissen increases and metastases arise in mouse models with human ovarian carcinomas. Social isolation also augments tumor mass. RNA interference specific for the human $\beta$-adrenoreceptor inhibits stress-enhanced tumor growth, whereas $\beta$-adrenoreceptor-negative ovarian cancer cell types do not respond to stress; thus, the stress-mediated increase in tumor burden requires $\beta$-adrenoreceptor expression on human ovarian cancer cells. The authors subsequently determine that $\beta$-adrenergic upregulation of cyclic AMP-protein kinase A signaling contributes to enhanced vascular endothelial growth factor (VEGF) mRNA and protein expression, thereby increasing tumor angiogenesis and subsequent growth. Administration of a VEGF-receptor 2 inhibitor and a monoclonal VEGF-specific antibody inhibits the effect of chronic stress on tumor burden. The study further suggests that $\beta$-adrenoreceptor inhibition might be useful for treatment of metastatic disease. (Nat. Med. 12, 939-944, 2006).

JWT

\section{Serogroup B meningococcus vaccine}

Vaccines have been available for decades against all but one serogroup, serogroup B, of Neisseria meningitides, a causal agent of meningitis and sepsis. Because serogroup B meningococcus has a capsular component identical to a human glycoprotein polysaccharide, creating a conventional glycoprotein conjugate vaccine has not been possible, although outer membrane vesicle vaccines with a strain coverage of only about $20 \%$ have been produced. Giuliani et al. have developed a universal vaccine effective against serogroup B strains using reverse vaccinology. In previous studies, the authors isolated 28 unique antigens and chose the five most promising vaccine candidates among these on the basis of whether they induced antibodies in rats and mice. They now combine these five antigens into one vaccine and test the antisera raised in mice for in vitro bactericidal activity against 85 strains of serogroup B meningococcus gathered from clinical isolates worldwide. The serum killed $78 \%$ of the strains tested, rising to almost $92 \%$ when an adjuvant suitable for human consumption, aluminum hydroxide, was combined with CpG oligonucleotides, and to $94 \%$ when the adjuvant MF59, approved for human use in Europe, was used. The researchers confirmed these results by inoculating infant rats and injecting them with one virulent meningococcus B strain. All of the control rats developed bacteremia, whereas none of those inoculated did. This work represents an important landmark in the translation of reverse vaccinology for meningoccoci into medical practice. (Proc. Natl. Acad. Sci. USA 103, 10834-10839, 2006)

$T M$

\section{Netrins promote vascular networks}

The observation that axonal pathfinding shares many similarities with formation of vascular networks has prompted recent insight into the roles of axon-guidance ligands and receptors in developmental angiogenesis. Wilson et al. show that netrins, one of four major classes of neural guidance cues that direct axonal growth during development, promote blood formation and reperfusion of ischemic tissue in two murine disease models. Vectors expressing netrins counteract hind-limb ischemia with comparable efficiency to those expressing vascular endothelial growth factor, and the unique therapeutic potential of netrins in treating diabetic complications resulting from both vasculopathy and neuropathy are revealed by their dual vascular and neural regenerative properties in the $d b / d b$ murine model of diabetes. These findings suggest that modulating netrin signaling offers promise for pro-angiogenic therapies (e.g., in ischemic ulcers, heart disease, stroke or diabetes) and in antiangiogenic treatments for cancer. (Science, published online 29 June 2006, DOI: 10.1126/science.1124704) 